\title{
Evaluation of elastic properties of submicrometer thin films using slanted finger interdigital transducers
}

\author{
Tsung-Tsong Wu, ${ }^{\text {a) }}$ Yung-Yu Chen, Guo-Tsai Huang, and Pei-Zen Chang \\ Institute of Applied Mechanics, National Taiwan University, Taipei, Taiwan
}

(Received 23 September 2004; accepted 7 January 2005; published online 22 March 2005)

\begin{abstract}
Accurate evaluation of film properties with thickness in the submicrometer range is very important throughout microelectronic industry as well as nano/microelectromechanical system industry. In this study, we developed a nondestructive evaluation technique, which is not only suitable for dielectric films but also valid for metallic films, to measure the elastic properties of submicrometer thin films. Firstly, we established the forward solution of the inverse evaluation and analyzed the dispersion of surface acoustic wave (SAW) in a $\mathrm{SiO}_{2} / \mathrm{YZ}-\mathrm{LiNbO}_{3}$ coated solid based on the effective permittivity approach. To measure the dispersion of SAW in the coated solid, a slanted finger interdigital transducer (SFIT) was employed to generate wide band SAW signals. The SFIT was designed by using the coupling of modes method to obtain the optimal frequency response. $\mathrm{SiO}_{2}$ films with submicrometer thickness were deposited on the piezoelectric $\mathrm{YZ}-\mathrm{LiNbO}_{3}$ substrate via the plasma-enhanced chemical-vapor deposition process. Pairs of the SFITs were also fabricated on the substrate. The measured frequency responses were then processed using the spectral analysis of surface wave to obtain the SAW dispersion in the coated solid. Finally, based on the forward solution and measured dispersion, we determined inversely the elastic properties of the $\mathrm{SiO}_{2}$ film through the use of the Simplex algorithm. The inversion result shows that the elastic properties of the submicrometer $\mathrm{SiO}_{2}$ film were measured successfully. We note that the results of this study provide an important basis for developing a SAW sensor which can be adopted to measure in situ film properties. (C) 2005 American Institute of Physics. [DOI: 10.1063/1.1865319]
\end{abstract}

\section{INTRODUCTION}

Thin films have been utilized widely throughout microelectronic industry as well as nano/microelectromechanical system (N/MEMS) industry. Their qualities have a great influence on the performance of devices, so the accurate evaluation of the film properties is necessary before designing and fabricating complex products. During the propagation of surface acoustic waves (SAWs), their acoustic energy concentrates within only one or two wavelengths under the surface. Since the SAW energy is confined closer to the surface with the increase of SAW frequency, the film properties affect more deeply the SAW propagation. Therefore, the highfrequency SAW technique is very suitable to measure the film properties, such as the elastic modulus.

Recently, several laser techniques were proposed to generate SAWs with frequency from megahertz to gigahertz, and further, adopted to evaluate the elastic properties of materials, such as the broadband laser technique ${ }^{1-3}$ and the laserinduced grating (LIG) method. ${ }^{4,5}$ Besides, in the 1990s, Hickernell and his coworkers ${ }^{6-9}$ conducted theoretical as well as experimental studies on the property measurements of a variety of thin films using SAW devices. They utilized the SAW device with split interdigital transducers to generate surface acoustic waves from under $100 \mathrm{MHz}$ to over $1 \mathrm{GHz}$. The frequency response of the SAW device was measured by a network analyzer to obtain SAW dispersion. We note that the harmonic resonance modes must be identified carefully

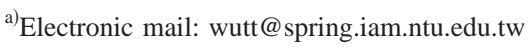

from the measured frequency response. The independent elastic constants of the measured films were determined inversely by a least-squares fit of the theoretical velocity dispersion curve to the measured one. However, this method can only be used to evaluate nonconductive materials. In addition, automatic determination of the various harmonic resonance modes is rather complicated.

The objective of this paper is to develop a nondestructive evaluation technique, not only suitable for dielectric but also valid for metallic films, to measure the elastic properties of submicrometer thin films. In the following, we present the theoretical analysis of the SAW dispersion in a coated piezoelectric medium. The design and fabrication of a thin-film SAW sensor with slanted finger interdigital transducers (SFITs) are described. Finally, we adopted the Simplex algorithm to recover the elastic properties of $\mathrm{SiO}_{2}$ film.

\section{DISPERSION OF SAW IN A COATED PIEZOELECTRIC MEDIUM}

The effective permittivity ${ }^{10}$ relates the surface potential and the charge density at the interface between the vacuum and the coated solid. In a plot of the effective permittivity of SAW as a function of phase velocity, the zeros correspond to the surface wave solution for a free surface, since the charge density is zero. The poles indicate the surface wave solution for a metallized surface, since it gives a finite charge density at zero electric potential. Therefore, in the following, we utilized the formulation for the effective permittivity based on the matrix method ${ }^{11-13}$ to calculate the SAW dispersion of 


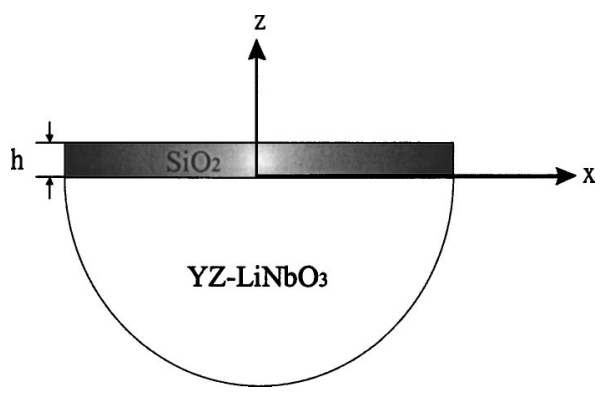

FIG. 1. Coordinate of a $\mathrm{SiO}_{2} / \mathrm{YZ}-\mathrm{LiNbO}_{3}$ coated solid in contact with a vacuum.

a coated piezoelectric medium. The relative material constants used in our calculations are listed in Ref. 14.

Shown in Fig. 1 is a $\mathrm{SiO}_{2} / \mathrm{LiNbO}_{3}$ coated solid in contact with a vacuum, where $h$ is the thickness of the $\mathrm{SiO}_{2}$ film. Figure 2 shows the dispersion curves of the fundamental surface wave mode in this coated solid. The phase velocity of the fundamental surface wave mode increases from Rayleigh wave velocity of $\mathrm{YZ}-\mathrm{LiNbO}_{3}$ with the increase of SAW frequency, and finally approaches to Rayleigh wave velocity of the $\mathrm{SiO}_{2}$ film. It is worth noting that the thicker the thickness of $\mathrm{SiO}_{2}$, the steeper the slopes of the dispersion curve. In addition, the differences of the dispersion curves for different $\mathrm{SiO}_{2}$ film thicknesses become larger with the increase of SAW frequency. In other words, the sensitivity of a thin-film SAW sensor is better in the high-frequency range.

\section{DESIGN AND FABRICATION OF A THIN-FILM SAW SENSOR}

\section{A. Design of a thin-film SAW sensor}

As shown in Fig. 3, we propose a design of a SAW sensor for measuring the elastic properties of thin films. In Fig. 3, the slanted finger interdigital transducer located on the left side is used for exciting the signal of surface acoustic waves, while the SFITs on the right side are used as receivers. The measured $\mathrm{SiO}_{2}$ film is located on the middle between one of the two SFIT pairs. The advantage of using the SFIT is that a very wide frequency bandwidth can be obtained to enhance greatly the inversion accuracy.

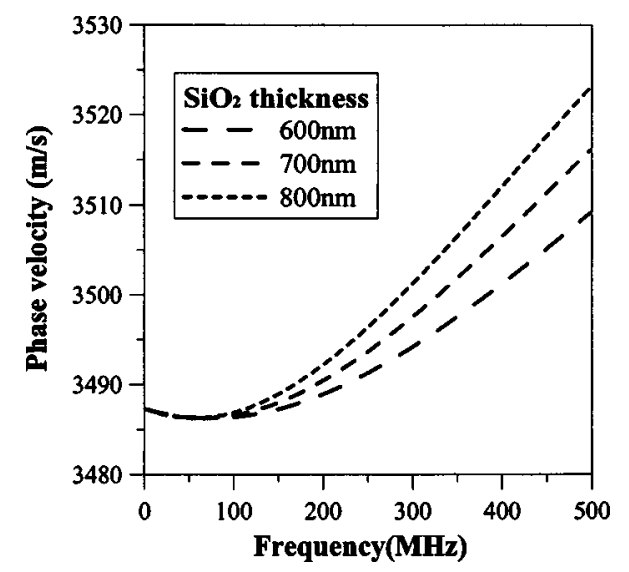

FIG. 2. Phase velocity dispersion as a function of frequency for the $\mathrm{SiO}_{2} / \mathrm{YZ}-\mathrm{LiNbO}_{3}$ coated solid.

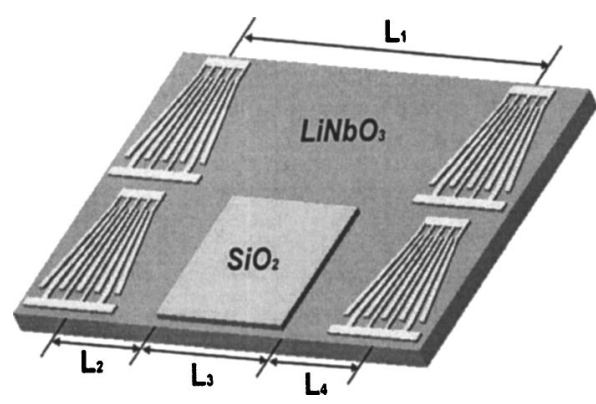

FIG. 3. Scheme of the thin-film SAW sensor.

In the following, we derive the formula for the phase velocity of surface acoustic wave in a $\mathrm{SiO}_{2} / \mathrm{YZ}-\mathrm{LiNbO}_{3}$ coated solid according to the spectral analysis of surface wave. ${ }^{15}$ From Fig. 3 , the phase difference $\phi_{1}$ induced by Rayleigh wave traveling through path $L_{1}$ is given by

$$
\phi_{1}=2 \pi f \frac{L_{1}}{V_{1}},
$$

where $V_{1}$ is the phase velocity of Rayleigh wave on $\mathrm{YZ}-\mathrm{LiNbO}_{3}$. The phase difference $\phi_{2}$ induced by the surface acoustic wave traveling through path $L_{2}+L_{3}+L_{4}$ is expressed as

$$
\phi_{2}=2 \pi f \frac{L_{2}}{V_{1}}+2 \pi f \frac{L_{3}}{V_{2}}+2 \pi f \frac{L_{4}}{V_{1}},
$$

where $V_{2}$ is the phase velocity of SAW on the $\mathrm{SiO}_{2} / \mathrm{YZ}-\mathrm{LiNbO}_{3}$ coated solid. Let $L_{1}$ be equal to $L_{2}+L_{3}$ $+L_{4}$, the phase difference induced by surface acoustic waves between the two paths can easily be derived as

$$
\Delta \phi=\phi_{2}-\phi_{1}=2 \pi f \frac{L_{3}}{V_{2}}-2 \pi f \frac{L_{3}}{V_{1}}=2 \pi f L_{3}\left(\frac{1}{V_{2}}-\frac{1}{V_{1}}\right) .
$$

Therefore, the phase velocity of the SAW on the $\mathrm{SiO}_{2} / \mathrm{YZ}-\mathrm{LiNbO}_{3}$ coated solid can be rearranged as

$$
V_{2}=\frac{1}{\left(\frac{\Delta \phi}{2 \pi f L_{3}}+\frac{1}{V_{1}}\right)} .
$$

The phase velocity of Rayleigh wave in $\mathrm{YZ}-\mathrm{LiNbO}_{3}$ is a constant and is equal to $3487.32 \mathrm{~m} / \mathrm{s}$. The width of the $\mathrm{SiO}_{2}$ film $L_{3}$ is set to be $2 \mathrm{~mm}$. Therefore, once the phase difference $\Delta \phi$ between the two paths is measured, the dispersion relation between the phase velocity $\left(V_{2}\right)$ of SAW in the $\mathrm{SiO}_{2} / \mathrm{YZ}-\mathrm{LiNbO}_{3}$ coated solid and the SAW frequency $(f)$ can be obtained based on Eq. (4).

In Fig. 2, we find that the higher the center frequency of the SAW device, the better the sensitivity in measuring the film properties. However, the phases caused by surface waves propagating along the two paths are increasing with the increase of SAW frequency. If the starting frequency of the passband of the SFIT is too high, the true phase difference $\Delta \phi$ between the two paths cannot be measured. Therefore, in order to obtain this true phase difference, the starting frequency of the passband of the SFIT cannot be increased 


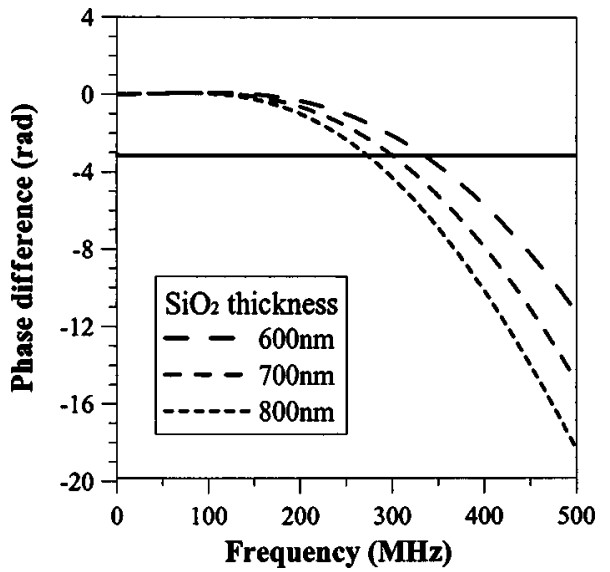

FIG. 4. Dispersion relation between phase difference and frequency for the $\mathrm{SiO}_{2} / \mathrm{YZ}-\mathrm{LiNbO}_{3}$ coated solid.

unlimitedly. In other words, the center frequency of the SFIT of the thin-film SAW sensor must be designed properly. Figure 4 shows the dispersion of the phase difference caused by surface waves propagating along the two different paths when $L_{3}$ is $2 \mathrm{~mm}$; the solid line means that the phase difference is equal to $-\pi$. We see that when the thickness of the $\mathrm{SiO}_{2}$ film is $800 \mathrm{~nm}$, the starting frequency of the passband of the SFIT must be lower than $280 \mathrm{MHz}$ to guarantee that a true phase difference can be measured. In addition, from Fig. 4 , it is worth noting that the thicker the $\mathrm{SiO}_{2}$ film thickness, the lower the starting frequency of the passband of SFIT.

\section{B. Fabrication of the thin-film SAW sensor}

The substrate of the thin-film SAW sensor adopted in this study is $\mathrm{YZ}-\mathrm{LiNbO}_{3}$ with $500 \mu \mathrm{m}$ in thickness. We deposited a $\mathrm{SiO}_{2}$ film on the $\mathrm{YZ}-\mathrm{LiNbO}_{3}$ wafer by a plasma-enhanced chemical-vapor deposition (PECVD) system. The source gas is a $233 \mathrm{~g} / \mathrm{s}$ oxygen mixed with a $140 \mathrm{~g} / \mathrm{s}$ tetraethylorthosilicate (TEOS) liquid. The substrate temperature is $350{ }^{\circ} \mathrm{C}$, and the background pressure is $53 \mathrm{~Pa}$. The rf power is $250 \mathrm{~W}$ which can lead to a deposition rate of $80 \mathrm{~nm} / \mathrm{min}$. The thicknesses of the $\mathrm{SiO}_{2}$ films used for the measurement are 500 and $820 \mathrm{~nm}$ which are measured by using a surface profiler. In order to enlarge the bandwidth of the wave signals, we employed the so-called slanted finger interdigital transducer as the SAW sources and receivers. SFIT SAW devices can provide a frequency response with small passband ripple, flat and wide passbands, large stop-band rejection, and steep cutoff characteristics. In

TABLE I. Geometry parameters of the slanted finger interdigital transducer.

$\begin{array}{cc}\lambda_{\min } \text { of SFIT } & 4 \mu \mathrm{m} \\ \lambda_{\max } \text { of SFIT } & 6.4 \mu \mathrm{m} \\ \text { Input pairs of SFIT } & 30 \\ \text { Output pairs of SFIT } & 20 \\ \text { Aperture } & 700 \mu \mathrm{m} \\ \text { Propagation distance } & 3326 \mu \mathrm{m} \\ \text { Maximum tilt angle } & 6^{\circ} \\ \text { Metallization ratio } & 0.5 \\ \text { Thickness of electrode } & 0.08 \mu \mathrm{m}\end{array}$

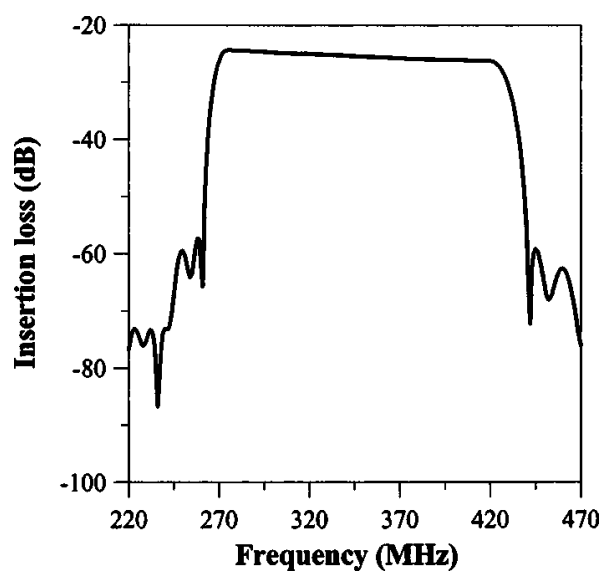

FIG. 5. Calculated frequency response of the thin-film SAW sensor with the SFIT.

this study, the coupling-of-mode (COM) $\operatorname{model}^{16}$ was used to analyze the frequency response of the SFIT SAW devices. The parameters of the SFIT are listed on Table I, while Fig. 5 shows the simulated frequency response of the SFIT.

\section{INVERSION OF THE ELASTIC PROPERTIES OF $\mathrm{SIO}_{2}$}

\section{A. Inversion scheme}

An error derivation function which defines the difference between the measured $\left(v_{m}\right)$ and the guessed $\left(v_{g}\right)$ phase velocities was expressed as

$$
e=\frac{\sum_{i=1}^{N}\left[v_{m}(i)-v_{g}(i)\right]^{2}}{\sum_{i=1}^{N}\left[v_{m}(i)\right]^{2}},
$$

where $i$ represents the discrete nondimensional wave number and $N$ is the number of data points. In the inversion process, initial guesses of the elastic constants and density of the $\mathrm{SiO}_{2}$ film are made first, then the computer program for calculating the phase velocity dispersion of surface waves in a coated solid is utilized to calculate the guessed phase veloci-

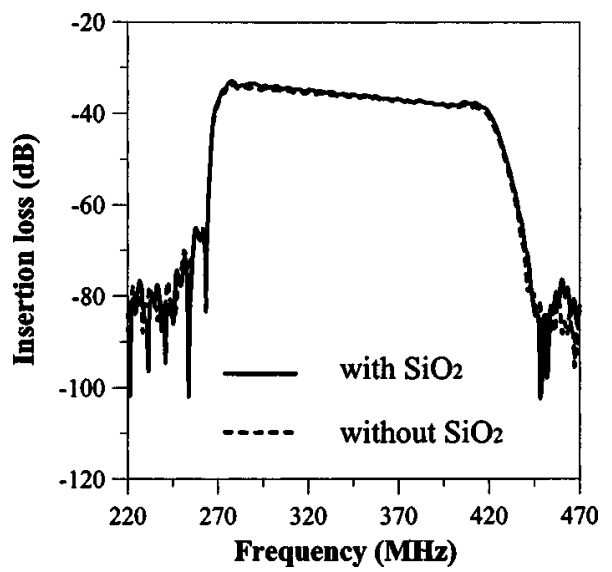

FIG. 6. Measured frequency responses of the SAW devices with and without $\mathrm{SiO}_{2}$ deposited on the delay line. 
TABLE II. Results of the inversion of density, $C_{11}, C_{44}$ when the thickness of $\mathrm{SiO}_{2}$ is $500 \mathrm{~nm}$.

\begin{tabular}{ccccccc}
\hline \hline $\begin{array}{c}\text { Guess } \\
d\end{array}$ & $\begin{array}{c}\text { Guess } \\
C_{11} \\
\left(\mathrm{Gg} / \mathrm{m}^{3}\right)\end{array}$ & $\begin{array}{c}\text { Guess } \\
C_{44} \\
(\mathrm{Gpa})\end{array}$ & $\begin{array}{c}\text { Result } \\
\left(\mathrm{kg} / \mathrm{m}^{3}\right)\end{array}$ & $\begin{array}{c}\text { Result } \\
C_{11} \\
(\mathrm{Gpa})\end{array}$ & $\begin{array}{c}\text { Result } \\
C_{44} \\
(\mathrm{Gpa})\end{array}$ & $\begin{array}{c}\text { Error } \\
\text { function } \\
\left(\times 10^{-8}\right)\end{array}$ \\
\hline \multicolumn{7}{c}{ Constraints } \\
$d=2180-2210 \mathrm{~kg} / \mathrm{m}^{3} C_{11}=70-80 \mathrm{GPa}$ & $C_{44}=20-30 \mathrm{GPa}$ \\
2180 & 70 & 30 & & & & \\
2190 & 73 & 26 & & & & \\
2200 & 76 & 23 & & & & \\
2210 & 80 & 20 & 2202 & 77.4 & 24.5 & 1.72 \\
2180 & 70 & 30 & & & & \\
2190 & 71 & 29 & & & & \\
2200 & 76 & 25 & & & & \\
2210 & 78 & 24 & 2202 & 76.4 & 24.8 & 1.90 \\
2180 & 71 & 28 & & & & \\
2190 & 72 & 27 & & & & \\
2200 & 78 & 24 & & & & \\
2210 & 80 & 23 & 2196 & 76.1 & 24.8 & 1.98 \\
\hline \hline
\end{tabular}

ties $\left(v_{g}\right)$. The value of the error derivation function can thus be obtained from Eq. (5). The true elastic constants and density of $\mathrm{SiO}_{2}$ are determined using the downhill Simplex method in this paper. A detailed flow chart of the Simplex method can be found in Ref. 17.

\section{B. Inversion of $\rho, C_{11}$, and $C_{44}$ of the $\mathrm{SiO}_{2}$ film}

A probe station and an Agilent 8714ES network analyzer are used to measure the frequency response of the SFIT SAW device. All the measurement work was done on wafer to avoid the parasitism effect caused by wire bounding and packaging. All the measured signals of frequency response are obtained by the time gating process to eliminate electromagnetic feedthrough and triple transit echo. Figure 6 shows the measured frequency responses of the SFIT pairs with and without $\mathrm{SiO}_{2}$ deposited on the delay lines when the $\mathrm{SiO}_{2}$ film is $500 \mathrm{~nm}$ in thickness. From the frequency responses shown in Fig. 6, it is noted that there is no obvious difference in the frequency responses of the two SFIT SAW devices, with and without $\mathrm{SiO}_{2}$ deposited on the delay lines, respectively. Attenuation of the SAW caused by the $\mathrm{SiO}_{2}$ film is not very obvious. In addition, the passbands of the wide band SAW devices are from 280 to $420 \mathrm{MHz}$, which is in good agreement with the simulated result shown in Fig. 5. Therefore, the range of the measured SAW dispersion is also from 280 to $420 \mathrm{MHz}$. The relation between phase difference and frequency $(\Delta \phi-f)$ can be calculated by subtracting the phase of the case without depositing $\mathrm{SiO}_{2}$ film deposited from that of the case with depositing $\mathrm{SiO}_{2}$ film deposited. Then, the dispersion curve of $\mathrm{SAW}$ in $\mathrm{SiO}_{2} / \mathrm{YZ}-\mathrm{LiNbO}_{3}$ coated solid can be obtained further according to Eq. (4).

In the following, to determine the elastic constants $C_{11}$, $C_{44}$ and the density $\rho$ of the $\mathrm{SiO}_{2}$ film simultaneously from the SAW dispersion, a set of four initial guessed points are needed in a three-dimensional (3D) space formed by $C_{11}$, $C_{44}$, and $\rho$. These initial guesses are constrained in the ranges $2180<\rho<2210 \mathrm{~kg} / \mathrm{m}^{3}, \quad 70<C_{11}<80 \mathrm{GPa}$, and $20<C_{44}$ $<30 \mathrm{GPa}$, which cover a wide range of the density and elastic constants of $\mathrm{SiO}_{2}$ materials. The thicknesses of the $\mathrm{SiO}_{2}$
TABLE III. Results of the inversion of density, $C_{11}, C_{44}$ when the thickness of $\mathrm{SiO}_{2}$ is $820 \mathrm{~nm}$.

\begin{tabular}{ccccccc}
\hline \hline $\begin{array}{c}\text { Guess } \\
d\end{array}$ & $\begin{array}{c}\text { Guess } \\
C_{11} \\
\left(\mathrm{Gg} / \mathrm{m}^{3}\right)\end{array}$ & $\begin{array}{c}\text { Guess } \\
C_{44} \\
(\mathrm{Gpa})\end{array}$ & $\begin{array}{c}\text { Result } \\
\left(\mathrm{kg} / \mathrm{m}^{3}\right)\end{array}$ & $\begin{array}{c}\text { Result } \\
C_{11} \\
(\mathrm{Gpa})\end{array}$ & $\begin{array}{c}\text { Result } \\
C_{44} \\
(\mathrm{Gpa})\end{array}$ & $\begin{array}{c}\text { Error } \\
\text { function } \\
\left(\times 10^{-7}\right)\end{array}$ \\
\hline \multicolumn{6}{c}{ Constraints } \\
$2=2180-2210 \mathrm{~kg} / \mathrm{m}^{3}$ & $C_{11}=70-80 \mathrm{GPa}$ & $C_{44}=20-30 \mathrm{GPa}$ \\
2180 & 70 & 30 & & & & \\
2190 & 71 & 29 & & & & \\
2200 & 76 & 25 & & & & \\
2210 & 78 & 24 & 2203 & 76.5 & 24.8 & 1.17 \\
2180 & 71 & 28 & & & & \\
2190 & 72 & 27 & & & & \\
2200 & 75 & 25 & & & & \\
2210 & 78 & 24 & 2202 & 75.7 & 25.3 & 1.17 \\
2180 & 69 & 30 & & & & \\
2190 & 74 & 26 & & & & \\
2200 & 76 & 24 & & & & \\
2210 & 78 & 22 & 2198 & 75.0 & 25.3 & 1.17 \\
\hline \hline
\end{tabular}

films are measured using a surface profiler as 500 and $820 \mathrm{~nm}$. In this study, 140 measured phase velocities for frequency between 280 and $420 \mathrm{MHz}$ are adopted in the inversion process. The inversion results of the density $\rho$ and elastic constants $C_{11}$ and $C_{44}$ of the $\mathrm{SiO}_{2}$ films with the thicknesses of 500 and $820 \mathrm{~nm}$ are shown in Tables II and III, respectively.

The values shown as follows are the mean values of the recovered density $\rho$ and elastic constants $C_{11}$ and $C_{44}$ for the two film thicknesses:

(1) $500 \mathrm{~nm}: \rho=2200 \pm 3 \mathrm{~kg} / \mathrm{m}^{3}, \quad C_{11}=76.6 \pm 0.6 \mathrm{GPa}$, and $C_{44}=24.7 \pm 0.1 \mathrm{GPa}$

(2) $820 \mathrm{~nm}: \rho=2201 \pm 2 \mathrm{~kg} / \mathrm{m}^{3}, \quad C_{11}=75.7 \pm 0.6 \mathrm{GP}$, and $C_{44}=25.1 \pm 0.2 \mathrm{GPa}$.

Figures 7 and 8 show the dispersion SAW phase velocity as a function of frequency for the $\mathrm{SiO}_{2}$ films with the thicknesses of 500 and $820 \mathrm{~nm}$. In Figs. 7 and 8, the open circles stand for the measured velocity dispersion data, and the dash lines are the theoretical dispersion curves for the 500- and $820-\mathrm{nm} \mathrm{SiO}_{2}$ films, while the solid lines are the fitted dispersion curves. The theoretical dispersion shown in the dash

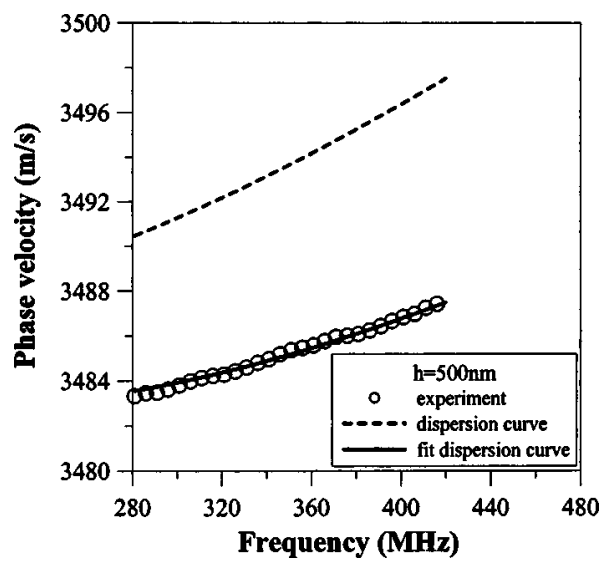

FIG. 7. Measured and calculated SAW velocity dispersion for 500 $-\mathrm{nm} \mathrm{SiO}_{2}$ on $\mathrm{LiNbO}_{3}$. 


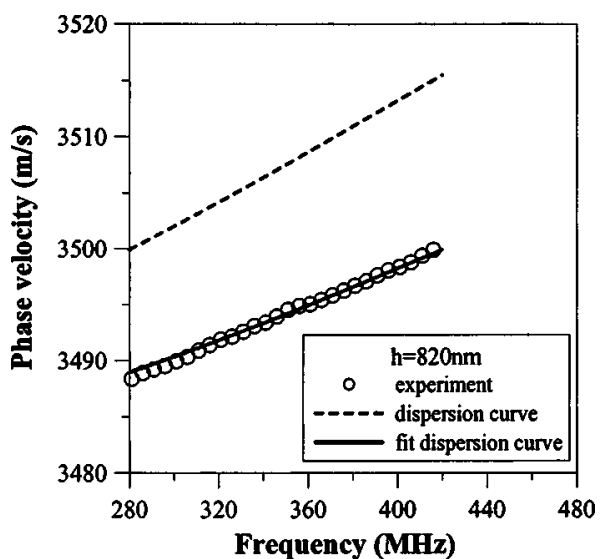

FIG. 8. Measured and calculated SAW velocity dispersion for 820 $-\mathrm{nm} \mathrm{SiO}{ }_{2}$ on $\mathrm{LiNbO}_{3}$.

lines were calculated based on the density and elastic constants for bulk fused silica with density of $2200 \mathrm{~kg} / \mathrm{m}^{3}$, the elastic constants as $C_{11}=78.5 \mathrm{GPa}$ and $C_{44}=31.2 \mathrm{GPa}$. Mismatch between the measured and the theoretical dispersion curve was found and comes from the differences between the properties of a bulk sample and those of a thin film. Figures 7 and 8 also show the comparison of the measured and the best-fit phase velocity dispersion based on the mean values of inversely determined density and elastic constants, and the results are in good agreements.

\section{CONCLUSION}

In this paper, to measure the SAW dispersion induced by the $\mathrm{SiO}_{2}$ film, a slanted finger interdigital transducer was designed properly and employed to generate wide band SAW signals. Based on the forward solution and measured dispersion of SAW in the coated solid, we determined inversely the elastic properties of the $\mathrm{SiO}_{2}$ film through the use of the Simplex algorithm. The recovered values of density $\rho$ and elastic constants $C_{11}$ and $C_{44}$ for submicrometer $\mathrm{SiO}_{2}$ films are very close to the elastic properties published in the literatures. ${ }^{9}$ It is worth noting that the wider the frequency range of the measured dispersion curve is, the more accurate the recovered elastic properties of the thin films. In summary, we have presented a method which utilized the SFIT SAW sensor to determine inversely the elastic properties of submicrometer thin films. In addition, results of this study provide an important basis for developing a SAW sensor which can be adopted to measure in situ film properties.

\section{ACKNOWLEDGMENT}

The authors thank the financial support of this research from the National Science Council of R.O.C. through the Grant No. NSC92-2212-E-002-001.

${ }^{1}$ D. Schneider, T. Schwarz, and B. Schultrich, Thin Solid Films 219, 92 (1992).

${ }^{2}$ T. T. Wu and Y. H. Liu, Ultrasonics 37, 23 (1999).

${ }^{3}$ X. Xiao, N. Hata, K. Yamada, and T. Kikkawa, Rev. Sci. Instrum. 74, 4539 (2002).

${ }^{4}$ J. A. Rogers and C. Case, Appl. Phys. Lett. 75, 865 (1999).

${ }^{5}$ Y. H. Liu, T. T. Wu, and C. K. Lee, J. Acoust. Soc. Am. 111, 2638 (2002).

${ }^{6}$ H. M. Liaw and F. S. Hickernell, IEEE Trans. Ultrason. Ferroelectr. Freq. Control 42, 404 (1995).

${ }^{7}$ T. S. Hickernell and F. S. Hickernell, IEEE Trans. Ultrason. Ferroelectr. Freq. Control 42, 410 (1995).

${ }^{8}$ I. S. Didenko, F. S. Hickernell, and N. F. Naumenko, IEEE Trans. Ultrason. Ferroelectr. Freq. Control 47, 179 (2000).

${ }^{9}$ F. S. Hickernell, T. S. Hickernell, and H. M. Liaw, Proc.-IEEE Ultrason. Symp. 381 (1994).

${ }^{10}$ T. T. Wu and Y. Y. Chen, IEEE Trans. Ultrason. Ferroelectr. Freq. Control 49, 142 (2002).

${ }^{11}$ A. H. Fahmy and E. L. Adler, Appl. Phys. Lett. 22, 495 (1973).

${ }^{12}$ K. A. Ingebrigtsen and A. Tonning, Phys. Rev. 184, 942 (1969).

${ }^{13}$ B. Honein, A. M. B. Braga, P. Barbone, and G. Herrmann, J. Intell. Mater. Syst. Struct. 2, 542 (1991).

${ }^{14}$ B. A. Auld, Acoustic Fields and Waves in Solids (Wiley, New York, 1976).

${ }^{15}$ S. Nazarian and M. Desai, J. Geotech. Eng. 119, 1094 (1992).

${ }^{16}$ T. T. Wu and C. M. Lin, J. Chin. Inst. Eng. 27973 (2004).

${ }^{17}$ J. F. Chai and T. T. Wu, J. Acoust. Soc. Am. 95, 3232 (1994). 